Tecnología y

Ciencias $\stackrel{\Xi}{\simeq}$ gua
2020, Instituto Mexicano de Tecnología del Agua

Open Access bajo la licencia CC BY-NC-SA 4.0

(https://creativecommons.org/licenses/by-nc-sa/4.0/)

Artículos

DOI: $10.24850 / j$-tyca-2020-02-04

\title{
Diseño gráfico para la materia orgánica y el tiempo de retención en lagunas facultativas
}

\section{Graphic design for organic matter and retention time in facultative lagoons}

Facundo Cortés-Martínez ${ }^{1}$, ORCID: 0000-0003-2189-3912

Alejandro Treviño-Cansino ${ }^{2}$, ORCID: 0000-0002-8451-4820

Ma. Aracelia Alcorta García ${ }^{3}$, ORCID: 0000-0002-9079-2771

Julio Gerardo Lozoya Vélez ${ }^{4}$

${ }^{1}$ Facultad de Ingeniería, Ciencias y Arquitectura, Universidad Juárez del Estado de Durango, Durango, Durango, México, fcortes@ujed.mx

${ }^{2}$ Facultad de Ingeniería, Ciencias y Arquitectura, Universidad Juárez del Estado de Durango, Durango, Durango, México, atrevinoc@ujed.mx

${ }^{3}$ Universidad Autónoma de Nuevo León, San Nicolás de los Garza, Nuevo León, México, maria.alcortagr@uanl.edu.mx

${ }^{4}$ Facultad de Ingeniería, Ciencias y Arquitectura, Universidad Juárez del Estado de Durango, Durango, Durango, México, gerardo_lovelez@ujed.mx

Autor para correspondencia: Facundo Cortés-Martínez, fcortes@ujed.mx 
Tecnología y

Ciencias $\stackrel{\Xi}{\unlhd}$ gua
2020, Instituto Mexicano de Tecnología del Agua

Open Access bajo la licencia CC BY-NC-SA 4.0

(https://creativecommons.org/licenses/by-nc-sa/4.0/)

\section{Resumen}

El presente trabajo proporciona dos gráficas con base en el diseño tradicional de una laguna facultativa. En la primera se establece un factor para el cálculo del tiempo de retención; en la segunda se determina la demanda bioquímica de oxígeno en el efluente del sistema, lo anterior con el fin de simplificar el diseño. Se aplicó el criterio del método gráfico, luego los valores obtenidos fueron verificados con los de la metodología tradicional. Se comprobó la simplificación en el dimensionamiento de una laguna facultativa. Es importante indicar que el presente estudio considera únicamente el análisis de la materia orgánica para el dimensionamiento.

Palabras clave: laguna facultativa, tiempo de retención, demanda bioquímica de oxígeno, diseño gráfico.

\section{Abstract}

The present paper provides two graphs based in the traditional design of a facultative pond. The first one establish a factor for the calculation of the retention time; the second one determines the biochemical oxygen demand in the effluent of the pond, the aforementioned in order to simplify the design. The graphic method was applied, later the data obtained were compared with the traditional method. It was demonstrated the simplification in the design of a facultative pond. It is important to mention that the present paper only considers the organic matter for the design. 
Tecnología y

Ciencias $\stackrel{\Xi}{\unlhd}$ gua
2020, Instituto Mexicano de Tecnología del Agua

Open Access bajo la licencia CC BY-NC-SA 4.0

(https://creativecommons.org/licenses/by-nc-sa/4.0/)

Keywords: Facultative lagoon, retention time, biochemical oxygen demand, graphic design.

Recibido: 23/04/2018

Aceptado: $15 / 07 / 2019$

\section{Introducción}

Los costos de mantenimiento y operación de las lagunas de estabilización son bajos, por esta razón estos sistemas de tratamiento son los más empleados; además, cumplen con la calidad del agua residual tratada para reutilizarla en la agricultura (Bracho, Aldana, García, \& Herrera, 1997; Mara, 2006; Gemitzi, Tsihrintzis, Christou, \& Petalas, 2007). Una desventaja importante en estos sistemas de tratamiento es que se necesitan grandes extensiones de terreno (Wood, Greenfield, Howes, Johns, \& Keller, 1995; Agunwamba, 2001; Mara, 2004; Kaya, Dilek, \& Gökcay, 2007; Naddafi et al., 2009). Los sistemas lagunares tienen como propósito disminuir la concentración de la materia orgánica, también conocida con la demanda bioquímica de oxígeno (DBO), en donde los procesos pueden ser tanto aerobios como anaerobios (Metcalf \& Eddy Inc., 1991). Según Rolim (2000), las lagunas de estabilización se clasifican en anaerobias, facultativas y de 
Tecnología y

Ciencias $\stackrel{\Xi}{\unlhd}$ gua
2020, Instituto Mexicano de Tecnología del Agua

Open Access bajo la licencia CC BY-NC-SA 4.0

(https://creativecommons.org/licenses/by-nc-sa/4.0/)

maduración, lo anterior en función del contenido de oxígeno. Las primeras tienen como característica principal la remoción de grandes cantidades de materia orgánica. De acuerdo con la Comisión Nacional del Agua (Conagua, 2007a) los estanques anaerobios son profundos (de 2.5 a 5.0 metros) y la eliminación de la DBO se lleva a cabo por medio de bacterias metanogénicas.

Las lagunas facultativas apoyan su funcionamiento por medio de bacterias, algas y la fotosíntesis, en donde la DBO se convierte en algas y productos inorgánicos. Una característica importante es que en la parte inferior prevalecen condiciones anaerobias; mientras que en la parte superior son aerobias (Rolim, 2000). Por otro lado, el viento favorece la mezcla, un reparto uniforme de la DBO, bacterias, algas y oxígeno disuelto. La consecuencia de lo indicado es un eficaz tratamiento (Conagua, 2007b). Sin embargo, estudios experimentales han demostrado que tiene más influencia el tamaño de las estructuras de entrada que la acción del viento, pues éste es muy variable en su dirección y velocidad (Shilton \& Harrison, 2003).

La función principal de los estanques de maduración es la eliminación de los coliformes fecales. El proceso que se establece es aerobio en toda la laguna y el oxígeno es generado por algas y la aireación superficial. Este tipo de lagunas son de poca profundidad (de 0.9 a 1.5 metros) (Metcalf \& Eddy Inc., 1991; Conagua, 2007a). De acuerdo con Heredia (1985) y Conagua (2007a), los coliformes fecales generan enfermedades, como cólera y tifoidea, entre otras. Los métodos de diseño que se aplican actualmente están basados en la carga máxima por unidad de área (Olukanni, 2011). 
Tecnología y

Ciencias $\stackrel{\Xi}{\triangleleft}$ gua
2020, Instituto Mexicano de Tecnología del Agua

Open Access bajo la licencia CC BY-NC-SA 4.0

(https://creativecommons.org/licenses/by-nc-sa/4.0/)

\section{De los diseños de sistemas lagunares con gráficas}

Swamee y Ojha (1991) reportaron una curva que representa el fenómeno de esfuerzo de la DBO como resultado de reacciones bioquímicas complejas entre el conjunto heterogéneo de biota y el sustrato orgánico complejo. Esta curva muestra una fase de retraso, seguida de una curva ascendente terminada en una meseta, y de nuevo una curva creciente de tipo saturante, que produce un efecto carbonatado de la DBO. Para el sustrato rico en compuestos nitrogenados, hay un aumento adicional en la curva de DBO, que satura en el valor de la DBO final de la segunda etapa. Ellis y Rodríguez (1995) determinaron ecuaciones de regresión múltiple para lagunas facultativas y de maduración, donde se relaciona la eliminación de la DBO y organismos coliformes fecales a una serie de parámetros ambientales y de otros tipos. Kehl, Wichern, Lübken y Horn (2009) presentaron un análisis estadístico para lagunas facultativas aireadas, facultativas y lagunas anaerobias por medio de método de análisis Monte Carlo. Presentan además algunos análisis estadísticos de los datos, como histogramas, gráficos de dispersión y tablas con los valores calculados para las variables de las lagunas de estabilización, a partir de los datos aleatorias de entrada. Morales, Domínguez y Mata (2016) publicaron un estudio estocástico: los autores introducen el factor dentro del modelo 
Tecnología y

Ciencias $\stackrel{\Xi}{\unlhd}$ gua
2020, Instituto Mexicano de Tecnología del Agua

Open Access bajo la licencia CC BY-NC-SA 4.0

(https://creativecommons.org/licenses/by-nc-sa/4.0/)

de lagunas facultativas por medio del método gráfico. Se aplica la gráfica con las isolíneas de fiabilidad obtenidas para el diseño estocástico del tratamiento mediante estanques con lemnáceas de los efluentes de las lagunas facultativas primarias de los municipios de Ranchuelo y Caibarién, en la provincia de Villa Clara, Cuba.

\section{Del diseño de lagunas facultativas con metodologías reducidas}

En la revisión bibliográfica realizada sólo se encontraron dos diseños de sistemas lagunares reducidos: a) manual simplificado para el diseño, operación y evaluación de lagunas de estabilización de aguas residuales de Collí, Rico, Rivas, Escalante y Luyendijk (1992), que considera, en términos generales, la calidad del agua residual en la entrada del sistema de lagunas, diseño, construcción, operación y mantenimiento; b) Treviño-Cansino y Cortés-Martínez (2016) reportaron otro criterio simplificado: método de diseño reducido para lagunas de estabilización, que estima como valores constantes la evaporación diaria ( $5 \mathrm{~mm} / \mathrm{día}$ ) y la profundidad $(1.5 \mathrm{~m})$. Además, es posible definir las dimensiones promedio de los taludes. Cabe mencionar que los dos criterios de diseño se basan en la metodología tradicional adoptada por la Conagua 
Tecnología y

Ciencias $\stackrel{?}{\square}$ Agua
2020, Instituto Mexicano de Tecnología del Agua

Open Access bajo la licencia CC BY-NC-SA 4.0

(https://creativecommons.org/licenses/by-nc-sa/4.0/)

(2007a). Se presentan las ecuaciones definidas para el último diseño exclusivamente de la laguna facultativa.

Volumen:

$V=\beta Q_{i}$

Donde:

$\beta=\left(\frac{z D B O_{i 10}}{\lambda_{s}}\right)$

Área superficial:

$A_{\text {superior }}=B_{\text {superior }} * L_{\text {superior }}$

Ancho superior:

$B_{\text {superior }}=\varepsilon Q_{i}+1.5 *$ pendiente

Longitud superior:

$L_{\text {Superior }}=3_{\varepsilon} Q_{i}+1.5 *$ pendiente 
Tecnología y

Ciencias $\stackrel{?}{\square}$ Agua
2020, Instituto Mexicano de Tecnología del Agua

Open Access bajo la licencia CC BY-NC-SA 4.0

(https://creativecommons.org/licenses/by-nc-sa/4.0/)

Donde $\varepsilon$ es un factor para el cálculo de las dimensiones de la laguna que depende de la temperatura.

Gasto en el efluente:

$Q_{e}=\omega Q_{i}-0.03 \varepsilon Q_{i} *$ pendiente $-0.01125 *$ pendiente $^{\wedge} 2$

Donde $\omega$ es un factor para simplificar el cálculo del gasto en el efluente.

Tiempo de retención hidráulico:

$O_{f}=\beta$

Número de coliformes fecales:

$\frac{N_{f}}{N_{o}}=\varphi \mathrm{N}_{i}\left(\frac{Q_{i}}{Q_{e}}\right)$

Donde $\varphi$ es un factor que depende de la relación largo-ancho, temperatura, y de la constante adimensional (a) y del factor de 
Tecnología y

Ciencias ฐ̃gua
2020, Instituto Mexicano de Tecnología del Agua

Open Access bajo la licencia CC BY-NC-SA 4.0

(https://creativecommons.org/licenses/by-nc-sa/4.0/)

dispersión $(d)$. Lo anterior para realizar el cálculo de los coliformes en el efluente de la laguna.

DBO en el efluente corregido por evaporación:

$$
D B O_{e}=\tau D B O_{i}\left(\frac{Q_{i}}{Q_{e}}\right)
$$

Donde $\tau$ es un factor para el cálculo de la DBO en el efluente que depende del tiempo de retención (0) y de la constante de decaimiento. El objetivo del presente trabajo es determinar dos gráficas con base en el diseño tradicional de una laguna facultativa. La primera tiene como propósito establecer un factor para el cálculo del tiempo de retención; mientras que la segunda determina la demanda bioquímica de oxígeno en el efluente de un sistema de tratamiento con lagunas de estabilización. La aportación del presente estudio es la deducción de un método gráfico para el dimensionamiento de una laguna facultativa, a partir del método reducido propuesto por Treviño-Cansiño y CortésMartínez (2016). Una ventaja importante con el criterio presentado es la simplificación, rapidez y facilidad para calcular el dimensionamiento de una laguna facultativa.

\section{Materiales y métodos}


El texto consultado para el diseño fue "Paquetes tecnológicos para el tratamiento de excretas y aguas residuales en comunidades rurales" (Conagua, 2007a).

\section{Nomenclatura para el diseño de la laguna facultativa}

C.O. = carga orgánica.

$Q_{i}=$ caudal en el influente de la laguna $\left(\mathrm{m}^{3} /\right.$ día $)$.

$D B O_{i}=$ concentración de la demanda bioquímica de oxígeno en el influente $(\mathrm{mg} / \mathrm{l})$.

1000 = factor de conversión.

$\lambda_{v}=$ carga orgánica superficial $\left(g D B O_{5} \mathrm{~m}^{3} /\right.$ día $)$.

$\mathrm{T}=$ temperatura mínima media mensual del aire $\left({ }^{\circ} \mathrm{C}\right)$.

$L_{i}=$ concentración de $D B O_{5}$ en el influente de la laguna $(\mathrm{mg} / \mathrm{l})$.

$A_{f}=$ área en $\mathrm{m}^{2}$.

$Q_{\text {med }}=$ caudal en el influente en $\mathrm{m}^{3} /$ día .

$\mathrm{Z}=$ profundidad en $\mathrm{m}$.

$V=$ volumen de la laguna $\left(\mathrm{m}^{3}\right)$. 
Tecnología y

Ciencias $\stackrel{\Xi}{\widetilde{v}}$ gua
2020, Instituto Mexicano de Tecnología del Agua

Open Access bajo la licencia CC BY-NC-SA 4.0

(https://creativecommons.org/licenses/by-nc-sa/4.0/)

$O_{f}=$ tiempo medio de retención hidráulico, laguna facultativa (días).

$X=$ relación entre longitud y anchura.

Bprom = ancho promedio de la laguna en $\mathrm{m}$.

Lprom = longitud promedio de la laguna en $\mathrm{m}$.

Bsup $=$ ancho superior en $\mathrm{m}$.

Lsup $=$ largo superior en $\mathrm{m}$.

Asup $=$ área superficial en $\mathrm{m}^{2}$.

$Q_{e}=$ caudal en el efluente de la laguna ( $\mathrm{m}^{3} /$ día).

e = evaporación ( $\mathrm{mm} /$ día $)$.

$d=$ factor de dispersión adimensional.

$a=$ constante adimensional.

$N_{e}=$ coliformes fecales corregidos por evaporación en el efluente de la laguna (NMP 100/ml).

$\mathrm{Ni}=$ coliformes fecales en el influente de la laguna (NMP 100/ml).

$N_{f} / N_{0}=$ número de coliformes fecales en el efluente (NMP $100 / \mathrm{ml}$ ).

$e=2.7182818$

$K f=$ constante de decaimiento a una temperatura en cualquier día.

$D B O_{e}=$ concentración de la $D B O_{i}$ en el efluente de la laguna $(\mathrm{mg} / \mathrm{l})$.

NMampF = número de mamparas en la laguna facultativa.

Talud $=$ relación de bordos $2: 1$. 
2020, Instituto Mexicano de Tecnología del Agua

Tecnología y

Ciencias $\stackrel{\Xi}{\varpi}$ gua

Open Access bajo la licencia CC BY-NC-SA 4.0

(https://creativecommons.org/licenses/by-nc-sa/4.0/)

\section{Diseño de laguna facultativa (flujo disperso. Método de Yáñez)}
a) Carga orgánica:
C.O. $=Q_{i}\left(D B O_{i}\right) / 1000$
b) Carga superficial de diseño:
$\lambda s=250(1.085)^{T-20}$
c) Área de la laguna facultativa:

$A_{f}=10 L_{i} Q_{m e d} / \lambda_{s}$

d) Volumen de la laguna:

$V=(A)(Z)$

e) Tiempo medio de retención hidráulico: 
2020, Instituto Mexicano de Tecnología del Agua

Tecnología y

Ciencias $\stackrel{\Xi}{\square}$ gua

Open Access bajo la licencia CC BY-NC-SA 4.0

(https://creativecommons.org/licenses/by-nc-sa/4.0/)

$O_{f}=V / Q_{i}$

f) Dimensionamiento. Relación largo ancho $X=3$ :

Bprom $=\sqrt{\frac{A f}{X}}$

Lprom $=\frac{A f}{B \text { Brom }}$

g) Para el ancho y largo:

Bsup $=$ Bprom $+(Z)($ Talud $)$

Lsup $=$ Lprom $+(Z)($ Talud $)$

h) Cálculo del área superficial:

Asup $=($ Bsup $)($ Lsup $)$

i) Gasto en el efluente: 
Tecnología y

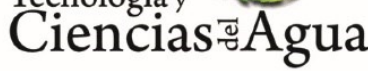

2020, Instituto Mexicano de Tecnología del Agua

Open Access bajo la licencia CC BY-NC-SA 4.0

(https://creativecommons.org/licenses/by-nc-sa/4.0/)

$Q_{e}=Q_{i}-0.001$ Asup

j) Remoción de coliformes fecales:

$X=3$ relación largo ancho

$d=\frac{X}{-0.26118+0.25392(X)+1.0136(X)^{2}}$

k) Coeficiente de reducción bacteriana:

$K b=0.841(1.075)^{T-20}$

I) Constante "a". Se determina a través de la fórmula:

$a=\sqrt{1+4 K_{b}} O_{f} d$

m) Coliformes fecales en el efluente de la laguna facultativa:

$\frac{N f}{N o}=\frac{4 a e^{(1-a / 2 d)}}{(1+a)^{2}} N_{i}$

n) Coliformes fecales corregidos por evaporación: 
Tecnología y

Ciencias $\stackrel{?}{\square}$ Agua
2020, Instituto Mexicano de Tecnología del Agua

Open Access bajo la licencia CC BY-NC-SA 4.0

(https://creativecommons.org/licenses/by-nc-sa/4.0/)

$N_{e}=\left(N_{e}\right)\left(Q_{i}\right) / Q_{e}$

ñ) Concentración de la DBO en el efluente de la laguna:

$K f=\frac{K f_{35}}{(1.085)^{35-T}}$

o) Concentración de la demanda bioquímica de oxígeno en el efluente de la laguna:

$D B O_{e f}=\frac{D B O_{i}}{K_{f} O_{f}+1}$

p) Eficiencia de remoción de la DBO:

$\%=\frac{\left(D B O_{i}-D B O_{e}\right)}{D B O_{i}} .100$

q) DBO corregida por evaporación:

$D B O_{e}=\left(D B O_{i}\right)\left(Q_{i}\right) / Q_{e}$ 
Tecnología y

Ciencias $\stackrel{\Xi}{\unlhd}$ gua
2020, Instituto Mexicano de Tecnología del Agua

Open Access bajo la licencia CC BY-NC-SA 4.0

(https://creativecommons.org/licenses/by-nc-sa/4.0/)

\section{Análisis matemático para el método gráfico de la materia orgánica y el tiempo de retención}

Dimensionamiento: tiempo de retención $(0)$.

Para determinar la gráfica del tiempo de retención se pretende realizar una función que tiene de variable independiente la temperatura. Para realizar lo anterior, se toma la Ecuación (2), que representa $\beta$ del método reducido publicado por Treviño-Cansiño y Cortés-Martínez (2016). Si se toma la Ecuación (7), que define el tiempo de retención en el método de diseño reducido, y se sustituye en la Ecuación (2), se tiene:

$O_{f}=\frac{Z * D B O_{i} 10}{\lambda_{s}}$

Por último se sustituye la fórmula de $\lambda s$ (Ecuación (11)) en la Ecuación (30) y se tiene:

$O_{f}=\frac{z * D B O_{i} 10}{250(1.085)^{T-20}}$

Según el método reducido, se fija como constante la profundidad ( $Z$ ) a $1.50 \mathrm{~m}$ y a diferencia de éste, se puede calcular el tiempo de retención para las diferentes concentraciones de $D B O_{e}$ indicadas por la 
Tecnología y

Ciencias $\stackrel{\Xi}{\simeq}$ gua
2020, Instituto Mexicano de Tecnología del Agua

Open Access bajo la licencia CC BY-NC-SA 4.0

(https://creativecommons.org/licenses/by-nc-sa/4.0/)

literatura (Metcalf \& Eddy Inc., 1991). Se considera la Expresión (26) del método de diseño reducido como constante de decaimiento a cualquier temperatura, y tomando la Ecuación (31) y expresándola en función del tiempo de retención y la $D B O_{i}$ se obtiene:

$\left(O / D B O_{i}\right)=\frac{1.5(10)}{250(1.085)^{T-20}}$

Luego, simplificando la Ecuación (32):

$\frac{O}{D B O_{i}}=\frac{0.30672276}{(1.085)^{T}}$

Graficando con Excel la Ecuación (33) se tiene la Figura 1:

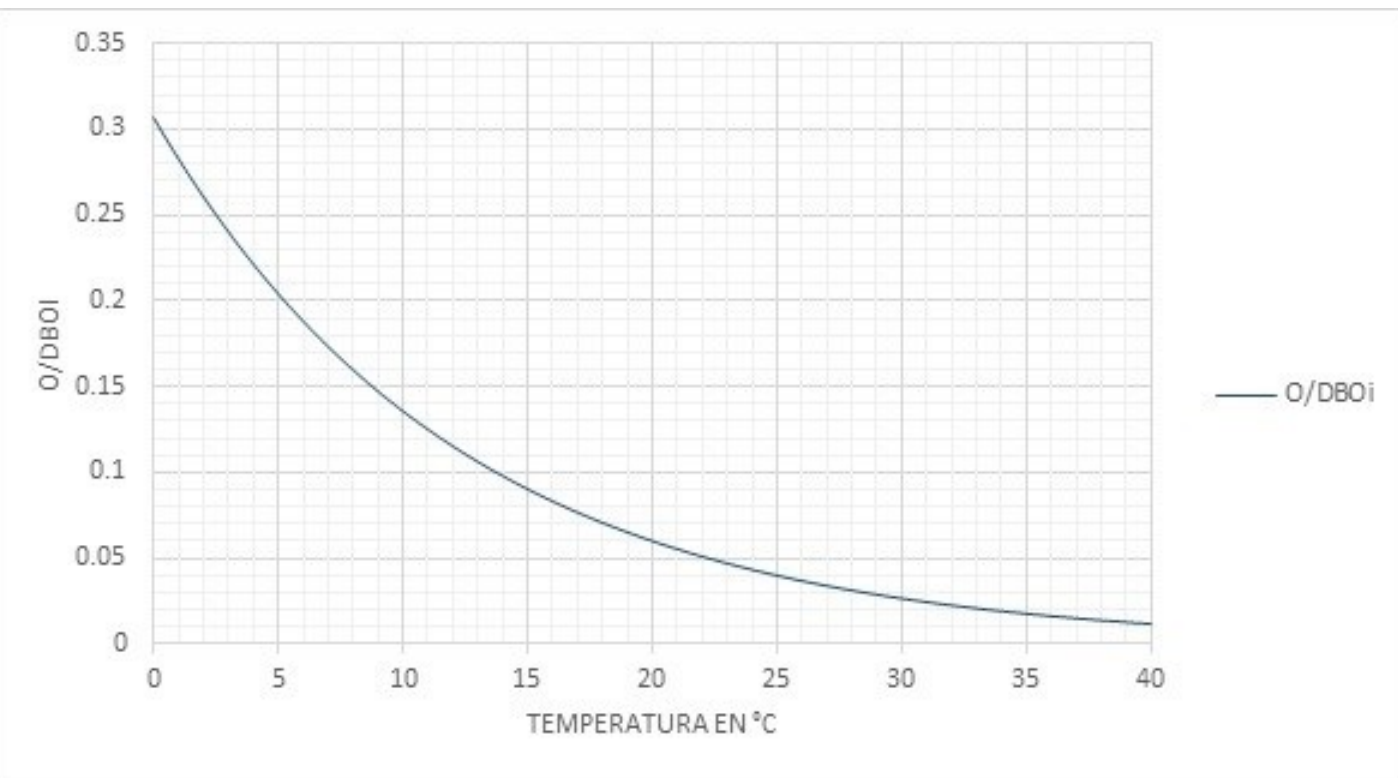


Tecnología y

Ciencias $\stackrel{\Xi}{\simeq}$ gua
2020, Instituto Mexicano de Tecnología del Agua

Open Access bajo la licencia CC BY-NC-SA 4.0

(https://creativecommons.org/licenses/by-nc-sa/4.0/)

Figura 1. Gráfica para el cálculo del tiempo de retención.

Para la $D B O$ en el efluente $\left(D B O_{e}\right)$, se toma como base la Fórmula (9) de la metodología y se elimina el factor de corrección por evaporación $\left(Q_{i} / Q_{e}\right)$, y resulta la Expresión (34):

$D B O_{e}=\tau * D B O_{i}$

Donde según Treviño-Cansiño y Cortés-Martínez (2016):

$\tau=\left(\frac{1}{K f O+1}\right)$

Se realiza la sustitución de la Ecuación (35) en la Ecuación (34) y se obtiene la Función (36):

$D B O_{e}=\left(\frac{1}{K f O+1}\right) * D B O_{i}$

En la Fórmula (36) se sustituye la constante de decaimiento $\mathrm{Kf}$ por la Expresión (26) de la metodología tradicional y el tiempo de retención $O$ por la Fórmula (31) y se tiene:

$D B O_{e}=\frac{1}{\left[\left(\frac{K f_{35}}{(1.085)^{35-T}}\right)\left(\frac{1.5(10) D B O_{i}}{250(1.085)^{T-20}}\right)+1\right.} * D B O_{i}$ 
Tecnología y

Ciencias $\stackrel{\Xi}{\widetilde{v}}$ gua
2020, Instituto Mexicano de Tecnología del Agua

Open Access bajo la licencia CC BY-NC-SA 4.0

(https://creativecommons.org/licenses/by-nc-sa/4.0/)

Posteriormente, se simplifica la Fórmula (37) y se sustituye la constante adimensional $k f_{35}=1.2$, y resulta la Expresión (38):

$D B O_{e}=\frac{D B O_{i}}{0.0211780721\left(D B O_{i}\right)+1}$

La Figura 2 muestra la gráfica para determinar la concentración de materia orgánica en la salida del sistema de tratamiento.

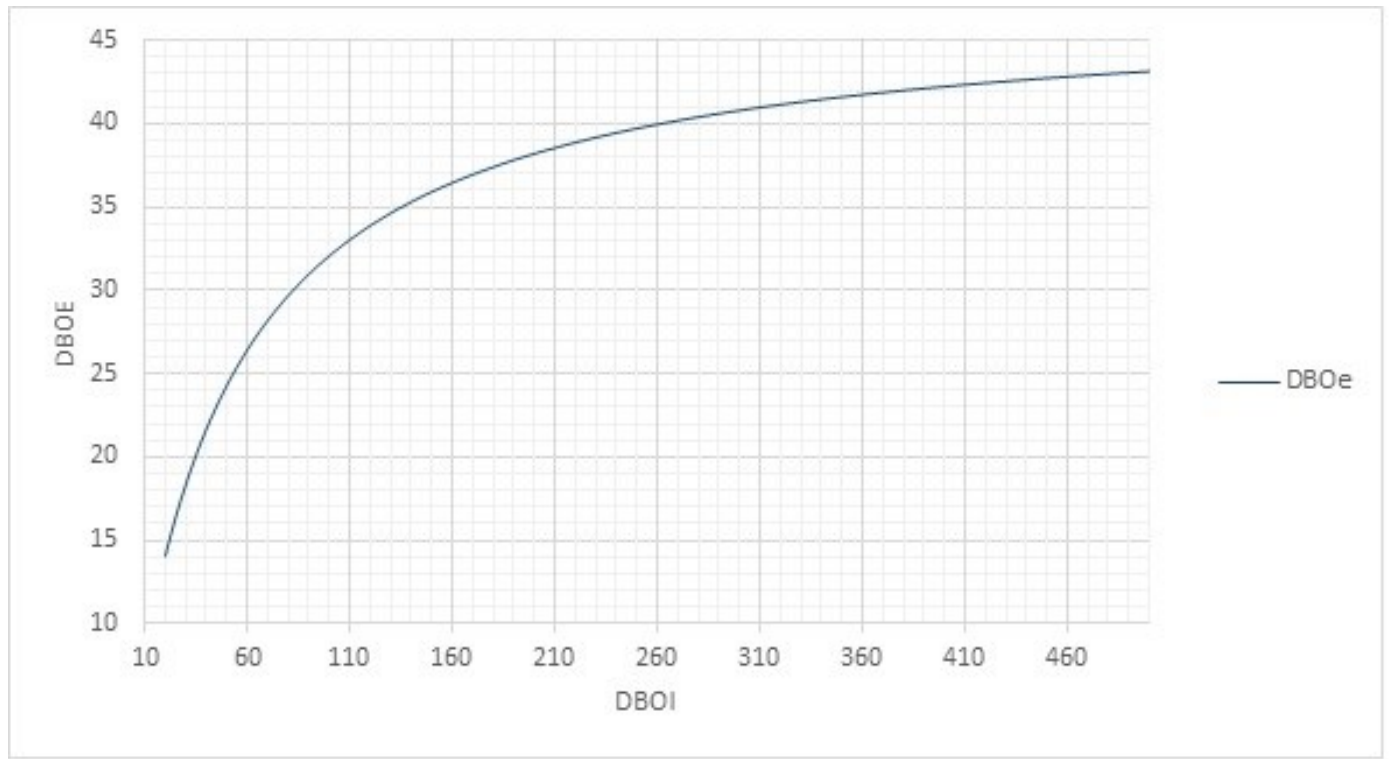

Figura 2. Gráfica para calcular la DBO en el efluente de la laguna facultativa. 
Tecnología y

Ciencias $\stackrel{?}{\square}$ Agua
2020, Instituto Mexicano de Tecnología del Agua

Open Access bajo la licencia CC BY-NC-SA 4.0

(https://creativecommons.org/licenses/by-nc-sa/4.0/)

\section{Aplicación del método gráfico}

Para la calcular el tiempo de retención, se obtiene el factor $\left(O_{f} / D B O i\right)$ de la Figura 2 y se multiplica por la $D B O i$ del caso en estudio:

$O_{f}=\left(O_{f} / D B O_{i}\right) * D B O_{i}$

A partir del tiempo de retención se puede deducir el volumen de la laguna al multiplicarlo por el gasto en el influente $(Q i)$ :

$V=O_{f} * Q_{i}$

El área de la laguna se obtiene al despejar la Fórmula (13) y fijando la profundidad $Z=1.50 \mathrm{~m}$ :

$A=\frac{V}{1.50}$

La demanda bioquímica de oxígeno en el efluente $\left(D B O_{e}\right)$ se obtiene de la Figura 2, con la $D B O_{i}$ como dato conocido. Para obtener el gasto en el efluente y las dimensiones de la laguna se aplican las 
Tecnología y

Ciencias $\stackrel{\Xi}{\widetilde{v}}$ gua
2020, Instituto Mexicano de Tecnología del Agua

Open Access bajo la licencia CC BY-NC-SA 4.0

(https://creativecommons.org/licenses/by-nc-sa/4.0/)

fórmulas del método tradicional (Ecuación (15), Ecuación (16), Ecuación (17), Ecuación (18) y Ecuación (20)).

\section{Ejemplo de aplicación}

Se solicita diseñar una laguna facultativa para una comunidad rural, utilizar la metodología tradicional y generar las gráficas para el tiempo de retención y la materia orgánica considerando los siguientes datos: Gasto $Q=231 \mathrm{~m}^{3} /$ día; demanda bioquímica de oxígeno en la entrada del sistema de tratamiento $D B O_{i}=220 \mathrm{mg} / \mathrm{l}$. Temperatura $T=11.80^{\circ} \mathrm{C}$ (Conagua, 2007b). Coliformes fecales en la entrada de la laguna: 10 $000000 \mathrm{NMP} / 100 \mathrm{ml}$ (Metcalf \& Eddy Inc., 1991). Finalmente, la evaporación con $5 \mathrm{~mm} /$ día.

\section{Resultados}

En la Tabla 1 se muestran los resultados del diseño.

Tabla 1. Resultados del diseño de una laguna facultativa con el criterio tradicional. 
Tecnología y

Ciencias 꽁ำ
2020, Instituto Mexicano de Tecnología del Agua

Open Access bajo la licencia CC BY-NC-SA 4.0

(https://creativecommons.org/licenses/by-nc-sa/4.0/)

\begin{tabular}{|c|c|c|c|c|c|c|c|c|c|c|c|c|}
\hline \multirow{2}{*}{\multicolumn{7}{|c|}{ Datos }} & \multicolumn{6}{|c|}{ Resultados } \\
\hline & & & & & & & \multicolumn{6}{|c|}{ Laguna facultativa } \\
\hline$Q_{i}$ & \multicolumn{2}{|l|}{$N f / N o_{i}$} & $\mathrm{DBO}_{\boldsymbol{i}}$ & $T$ & $\boldsymbol{Z}$ & $\boldsymbol{X}$ & \multicolumn{2}{|c|}{$\lambda_{s}$} & \multicolumn{2}{|c|}{$\boldsymbol{A f}$} & Vol. & $\boldsymbol{O}_{f}$ \\
\hline 231 & $\begin{array}{l}10000 \\
000\end{array}$ & \multicolumn{2}{|c|}{220} & 11.8 & 1.5 & 3.00 & \multicolumn{2}{|c|}{128.06} & \multicolumn{2}{|c|}{3968.43} & 5952.64 & 25.77 \\
\hline \multicolumn{13}{|c|}{ Resultados, laguna facultativa (continuación) } \\
\hline$d$ & $\boldsymbol{k} \boldsymbol{b}$ & \multicolumn{2}{|c|}{$a$} & $B_{\text {sup }}$ & $L_{\text {sup }}$ & \multicolumn{2}{|c|}{$Q_{e}$} & \multicolumn{2}{|c|}{$N f / N o$} & \multicolumn{2}{|c|}{$\overline{D B O_{e}}$} & Área \\
\hline 0.31 & 0.46 & \multicolumn{2}{|c|}{3.99} & 39.377 & 112.11 & \multicolumn{2}{|c|}{208.93} & \multicolumn{2}{|c|}{58391.01} & \multicolumn{2}{|c|}{42.98} & 4413.87 \\
\hline
\end{tabular}

\section{Diseño gráfico}

Se utiliza la Figura 1 y Figura 2 para obtener el factor $\left(O f / D B O_{i}\right)$ y la $D B O_{e}$, respectivamente. La Figura 3 y Figura 4 ilustran la aplicación al indicar la intersección con la curva. Luego se sigue el procedimiento indicado en el apartado de Aplicación del método gráfico: Ecuación (39), Ecuación (40) y Ecuación (41). 
Tecnología y

Ciencias $\cong$ Agua
2020, Instituto Mexicano de Tecnología del Agua

Open Access bajo la licencia CC BY-NC-SA 4.0

(https://creativecommons.org/licenses/by-nc-sa/4.0/)

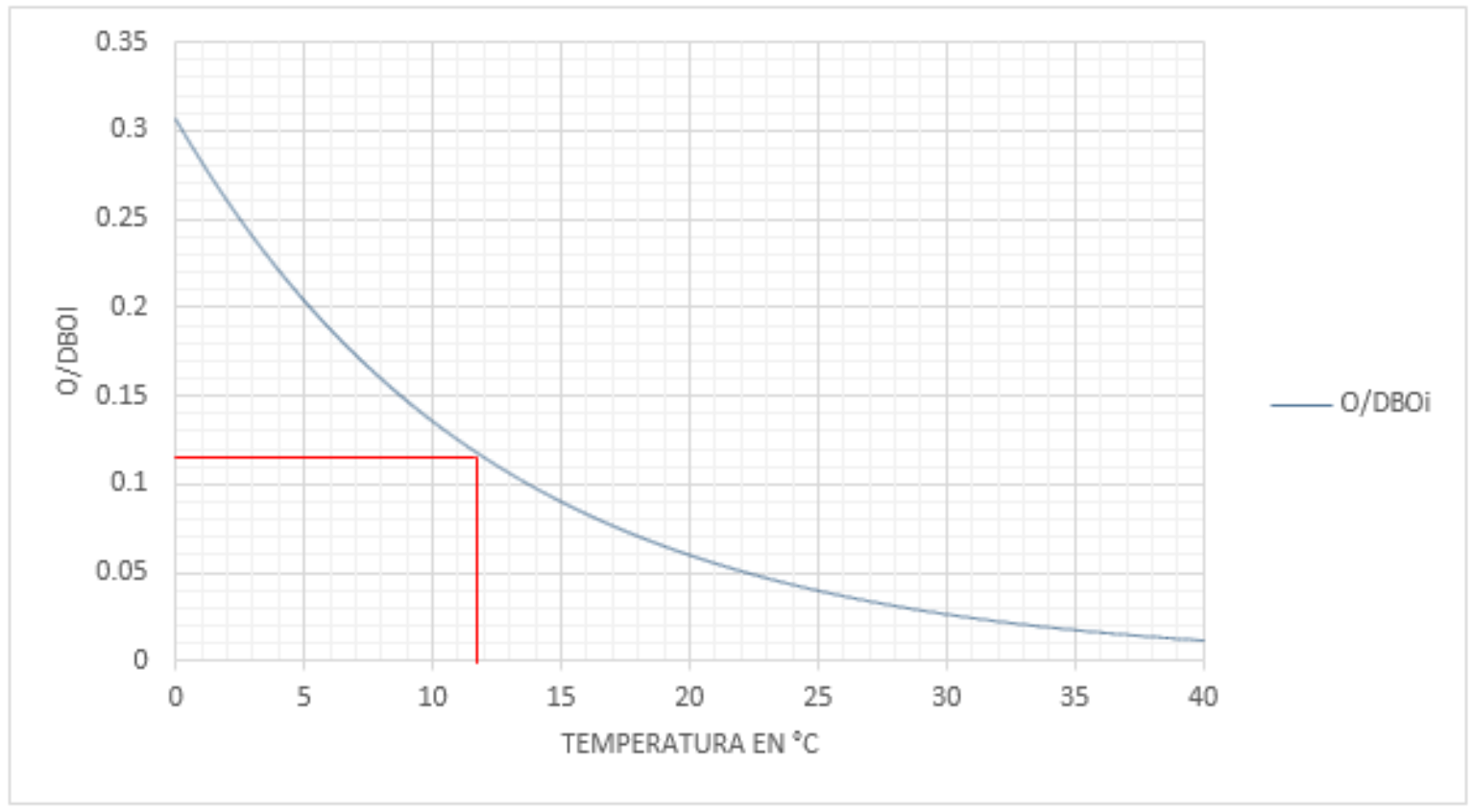

Figura 3. Ejemplo para el cálculo del tiempo de retención de una laguna facultativa.

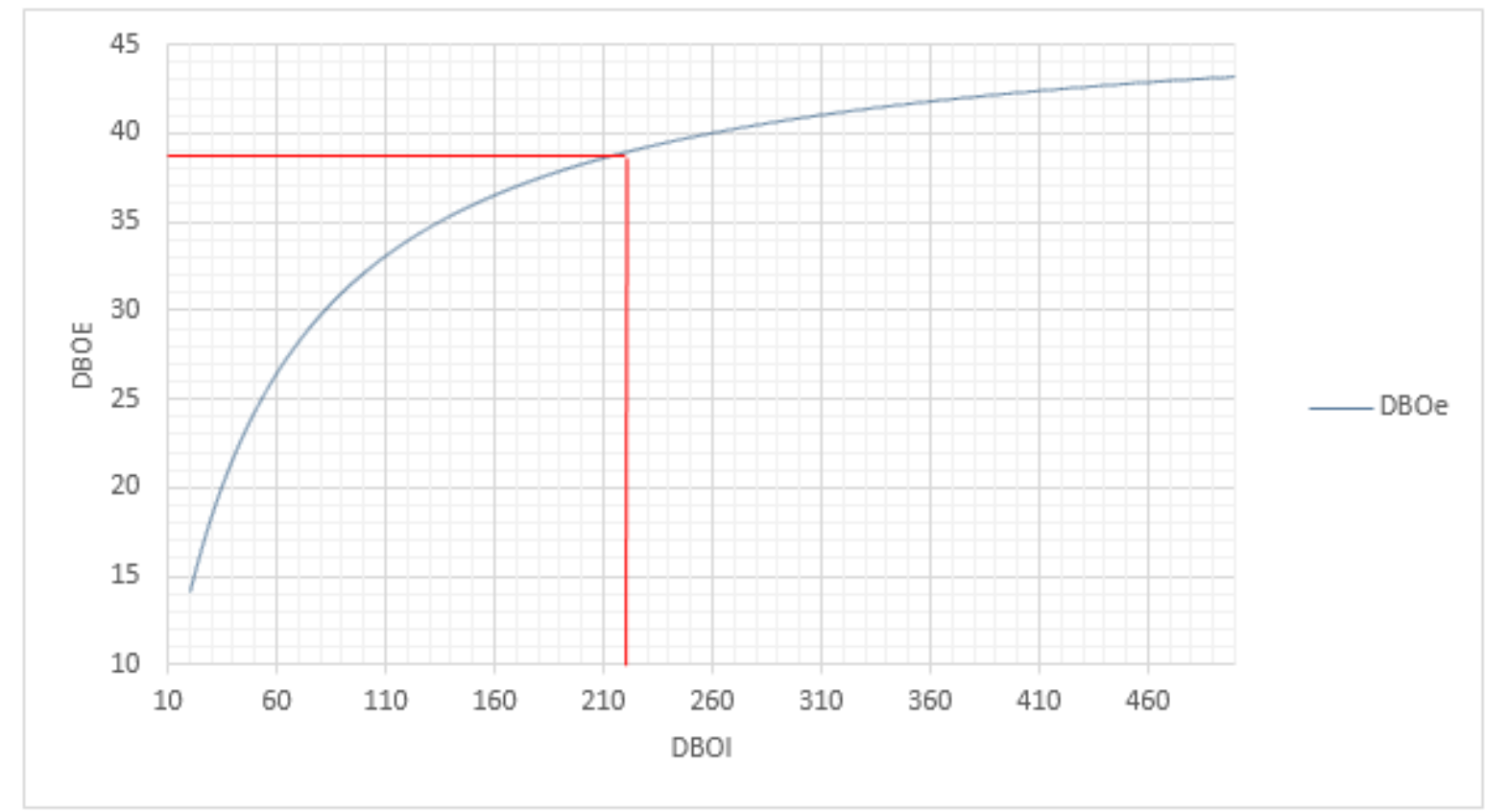


Ciencogiay

2020, Instituto Mexicano de Tecnología del Agua

Open Access bajo la licencia CC BY-NC-SA 4.0

(https://creativecommons.org/licenses/by-nc-sa/4.0/)

Figura 4. Ejemplo para calcular la DBO en el efluente de una laguna facultativa.

En la Tabla 2 se muestran los resultados del diseño gráfico.

Tabla 2. Resultados del diseño de laguna facultativa con el método gráfico

\begin{tabular}{|c|c|c|c|c|c|c|c|c|}
\hline \multicolumn{6}{|c|}{ Datos } & \multicolumn{3}{|c|}{ Resultados } \\
\hline$Q_{i}$ & $N f / N o_{i}$ & $D_{B O}$ & $T$ & $Z$ & $\boldsymbol{X}$ & $\mathrm{DBO}_{e}$ & $O / D B O_{i}$ & $\boldsymbol{O}_{f}$ \\
\hline 231 & 10000000 & 220 & 11.8 & 1.5 & 3.00 & 38.87 & 0.117 & 25.77 \\
\hline \multicolumn{9}{|c|}{ Resultados, laguna facultativa } \\
\hline$d$ & $\boldsymbol{k} \boldsymbol{b}$ & $a$ & $B_{\text {sup }}$ & $L_{\text {sup }}$ & $Q_{e}$ & $N f / N o$ & $D_{B O}$ & Área \\
\hline 0.31 & 0.46 & 3.99 & 39.37 & 112.11 & 208.93 & 58391.02 & 42.98 & 4413.87 \\
\hline
\end{tabular}

Como se observa en la Tabla 1 y Tabla 2, los resultados son similares: el mismo tiempo de retención de 25.77 días; demanda bioquímica de oxígeno corregida por evaporación de $42.98 \mathrm{mg} / \mathrm{l}$; área, 4 $413.87 \mathrm{~m}^{2}$; de igual forma las dimensiones. Cabe señalar que el método gráfico es más rápido y sencillo de calcular que el tradicional, por lo que puede utilizarse de forma confiable. Otra aplicación es para verificar los resultados obtenidos con la metodología tradicional; al respecto, Oliveira y Sperling (2011) reportaron que cuando los sistemas lagunares no cumplen con la calidad del agua residual tratada se debe a errores en su 
Tecnología y

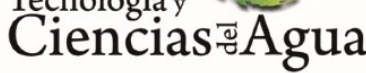

2020, Instituto Mexicano de Tecnología del Agua

Open Access bajo la licencia CC BY-NC-SA 4.0

(https://creativecommons.org/licenses/by-nc-sa/4.0/)

diseño, además de la operación. Ellis y Rodríguez (1995), Kehl et al. (2009), Swamee y Ojha (1991) presentaron distintos criterios de diseño con diferentes tipos de gráficas; mientras que en el presente estudio sólo se consideró el método tradicional de diseño adoptado por la Comisión Nacional del Agua y el Instituto Mexicano de Tecnología del Agua, pero disminuyendo las expresiones matemáticas a su mínima expresión posible, para luego elaborar las gráficas para el tiempo de retención y la remoción de la materia orgánica. Una ventaja del estudio propuesto es que de las 18 ecuaciones establecidas en el diseño tradicional de una laguna facultativa, se redujo a nueve. Las ecuaciones suprimidas por el método gráfico fueron las siguientes: (10), (11), (12), (13), (14), (26), (27), (28) y (29). Mientras que en el método de diseño reducido de Collí et al. (1992) reportaron 10 ecuaciones. La calidad del agua residual en el efluente de la laguna facultativa es la NOM-001SEMARNAT-1996, que indica los límites máximos de contaminantes que pueden descargarse en los cuerpos receptores; dicha norma fue publicada por el Diario Oficial de la Federación (DOF, 1997). Los límites máximos permisibles de materia orgánica para uso en riego agrícola que indica la citada norma es de $74 \mathrm{mg} / \mathrm{l}$ como promedio mensual; por lo tanto, la laguna facultativa cumple con la regulación, pues la concentración en la salida del estanque resultó de $42.98 \mathrm{mg} / \mathrm{l}$.

\section{De los coliformes fecales}


Tecnología y

Ciencias $₫$ Agua
2020, Instituto Mexicano de Tecnología del Agua

Open Access bajo la licencia CC BY-NC-SA 4.0

(https://creativecommons.org/licenses/by-nc-sa/4.0/)

La normatividad señala como límite máximo permisible 1000 NMP/100 $\mathrm{ml}$ como promedio diario. Los valores de coliformes fecales en el efluente del sistema resultaron por arriba del valor permitido, por lo que es recomendable el diseño de otra laguna a facultativa o de maduración (Rolim, 2000; Conagua, 2007b). Otra alternativa es el uso de mamparas o deflectores. Killani y Ogunrombi (1984); Pedahzur, Nasser, Dor, Fattal y Shuval (1993); Muttamara y Puetpaiboon (1997); Zanotelli, Medri, Belli-Filho, Perdomo y Costa (2002); Shilton y Harrison (2003); Sperling, Chernicharo, Soares y Zerbini (2003); Shilton y Mara (2005); Abbas, Nasr y Seif (2006); Cortés-Martínez, Treviño-Cansino, LuévanosRojas, Luévanos-Rojas y Uranga (2014a); Martínez, Cansino, García, Kalashnikov y Rojas (2014) reportaron que se incrementa la eliminación de contaminantes con el uso de mamparas. En la Figura 5 se muestran las dimensiones de la laguna facultativa calculadas con la metodología tradicional y con el diseño gráfico.

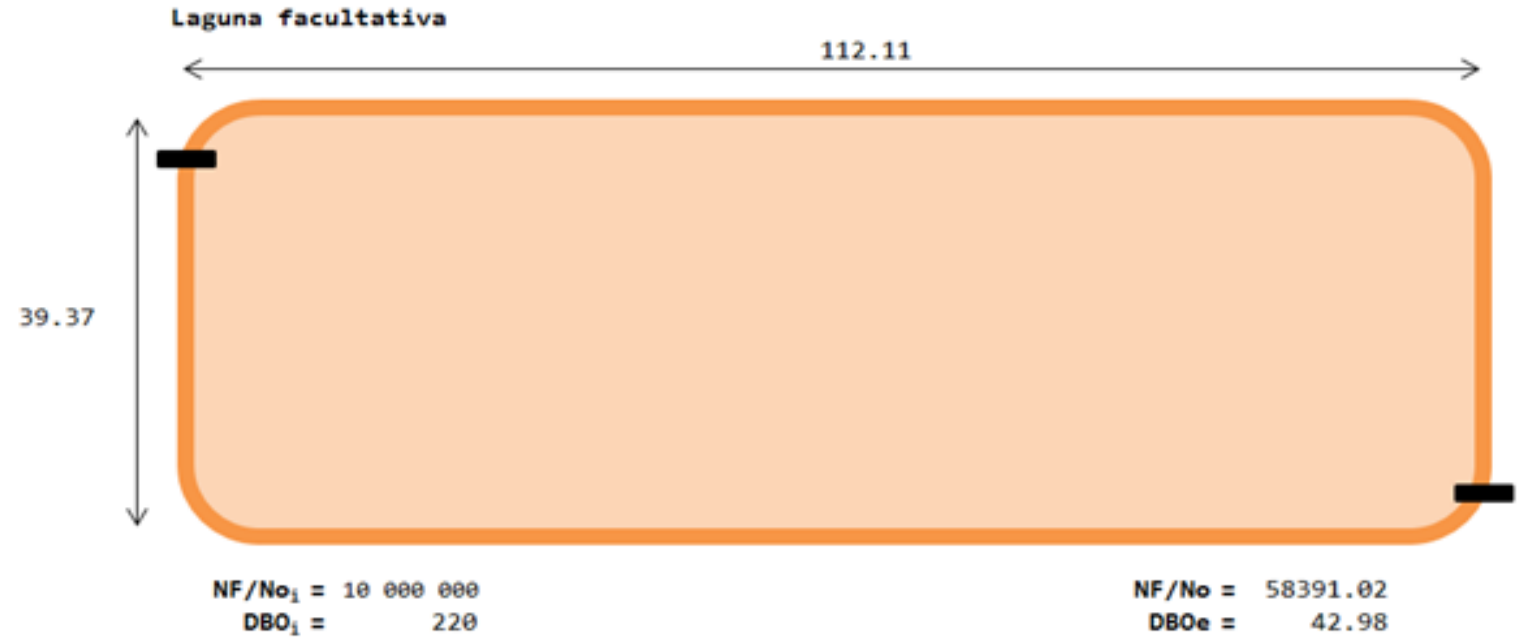


Tecnología y

Ciencias $\stackrel{\Xi}{\widetilde{v}}$ gua
2020, Instituto Mexicano de Tecnología del Agua

Open Access bajo la licencia CC BY-NC-SA 4.0

(https://creativecommons.org/licenses/by-nc-sa/4.0/)

Figura 5. Ilustración de las dimensiones de la laguna facultativa, aplicando ambas metodologías.

\section{Conclusiones}

De acuerdo con los objetivos planteados, se determinaron dos gráficas para el diseño de una laguna facultativa.

La simplificación en el diseño de la laguna facultativa es de consideración, ya que las ecuaciones disminuyeron el $50 \%$ aproximadamente.

Con el propósito de simplificar todavía más el diseño se recomienda llevar a cabo otro estudio, como trabajo a futuro, donde se consideren los coliformes fecales.

Es importante aclarar que el presente estudio refiere a un análisis matemático, por lo que la calidad del agua puede diferir del esperado, aunque también influyen la ubicación de entrada y salida de las estructuras, la geometría de las lagunas, la operación y el mantenimiento.

\section{Referencias}


Teçnología y

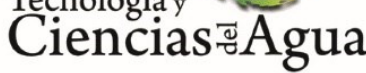

2020, Instituto Mexicano de Tecnología del Agua

Open Access bajo la licencia CC BY-NC-SA 4.0

(https://creativecommons.org/licenses/by-nc-sa/4.0/)

Abbas, H., Nasr, R., \& Seif, H. (2006). Study of waste stabilization pond geometry for wastewater treatment efficiency. Ecology Engineering, 28(1), 25-34. DOI:10.1016/j.ecoleng.2006.03.008

Agunwamba, J. C. (2001). Effect of tapering on the performance of waste stabilization ponds. Water Research, 35(5), 1191-1200. DOI: $10.1016 /$ S0043-1354(00)00391-2

Bracho, N., Aldana, G., García, C., \& Herrera, L. (1997). Análisis de las variables operacionales en la remoción de tratamiento de un sistema experimental de lagunas. Interciencias, 22(3), 138-143.

Conagua, Comisión Nacional del Agua. (2007a). Manual de diseño de agua potable, alcantarillado y saneamiento. Paquetes tecnológicos para el tratamiento de excretas y aguas residuales en comunidades rurales. Jiutepec, México: Comisión Nacional del Agua, Instituto Mexicano de Tecnología del Agua.

Conagua, Comisión Nacional del Agua. (2007b). Manual de diseño de agua potable, alcantarillado y saneamiento. Manual de diseño de lagunas de estabilización. Jiutepec, México: Comisión Nacional del Agua, Instituto Mexicano de Tecnología del Agua.

Collí, M. J., Rico, M. M., Rivas, H. A., Escalante, E. V. E., \& Luyendijk, R. (1992). Manual simplificado para el diseño, operación y evaluación de lagunas de estabilización de aguas residuales. Jiutepec, México: Comisión Nacional del Agua, Instituto Mexicano de Tecnología del Agua.

Cortés-Martínez, F., Treviño-Cansino, A., Luévanos-Rojas, A., LuévanosRojas, R., \& Uranga, S. A. C. (2014a). Función objetivo en el 
Tecnología y

Ciencias $\stackrel{\Xi}{\widetilde{v}}$ gua
2020, Instituto Mexicano de Tecnología del Agua

Open Access bajo la licencia CC BY-NC-SA 4.0

(https://creativecommons.org/licenses/by-nc-sa/4.0/)

diseño de la laguna facultativa (estudio de caso). Revista Mexicana de Ciencias Agrícolas, 5(3), 433-447.

DOF, Diario Oficial de la Federación. (1997). Norma Oficial Mexicana NOM-001-Semarnat-1996: que establece los límites máximos permisibles de contaminantes en las descargas de aguas residuales a los sistemas en aguas y bienes nacionales (30 pp.). México, DF, México: Diario Oficial de la Federación.

Ellis, K. V., \& Rodrigues, P. C. C. (1995). Multiple regression design equations for stabilization ponds. Water Research, 29(11), 25092519. DOI: $10.1016 / 0043-1354(95) 00081-u$

Gemitzi, A., Tsihrintzis, V. A., Christou, O., \& Petalas Ch. (2007). Use of GIS in siting stabilization pond facilities for domestic wastewater treatment. Journal of Environmental Management, 82(2), 155166. DOI: $10.1016 / j$.jenvman.2005.12.022

Heredia, D. M. D. (1985). Tecnología moderna para el tratamiento de aguas residuales. Ingeniería hidráulica en México, 1(1), 13-20.

Kaya, D., Dilek, F. B., \& Gökcay, C. F. (2007). Reuse of lagoon effluents in agriculture by post-treatment in a step feed dual treatment process. Desalination, 215(1-3), 29-36. DOI: 10.1016/j.desal.2006.11.013

Kehl, O., Wichern, M., Lübken, M., \& Horn, H. (2009). Analysis of design approaches for stabilization ponds under different boundary conditions. A comparison. Ecological Engineering, 35(8), 11171128. 
Tecnología y

Ciencias $\stackrel{\Xi}{\widetilde{v}}$ gua
2020, Instituto Mexicano de Tecnología del Agua

Open Access bajo la licencia CC BY-NC-SA 4.0

(https://creativecommons.org/licenses/by-nc-sa/4.0/)

Killani, J. S., \& Ogunrombi, J. A. (1984). Effects of baffles on the performance of model waste stabilization ponds. Water Research, 18(8), 941-944. DOI: 10.1016/0043-1354(84)90243-4

Mara, D. D. (2004). Domestic wastewater treatment in developing countries. Recuperado de https://www.routledge.com/DomesticWastewater-Treatment-in-DevelopingCountries/Mara/p/book/9781844070190

Mara, D. D. (2006). Constructed wetlands and waste stabilization ponds for small rural communities in the United Kingdom: A comparison of land area requirements, performance and costs. Environmental Technology, 27(7), 753-757.

Martínez, F. C., Cansino, A. T., García, M. A. A., Kalashnikov, V., \& Rojas, R. L. (2014). Mathematical analysis for the optimization of a design in a facultative pond: Indicator organism and organic matter. Mathematical Problems in Engineering, 2014, 1-12. DOI: $10.1155 / 2014 / 652509$

Metcalf \& Eddy Inc. (1991). Wastewater Engineering. Treatment, disposal, reuse. New York, USA: McGraw-Hill.

Morales, R. S., Domínguez, E. R., \& Mata, M. M. (2016). Procedimiento gráfico para la implementación del diseño estocástico —bajo condiciones de incertidumbre y variabilidad- de unidades naturales de tratamiento de aguas residuales. Afinidad, 73(575), 219-225. 
Tecnología y

Ciencias $\stackrel{\Xi}{\widetilde{v}}$ gua
2020, Instituto Mexicano de Tecnología del Agua

Open Access bajo la licencia CC BY-NC-SA 4.0

(https://creativecommons.org/licenses/by-nc-sa/4.0/)

Muttamara, S., \& Puetpaiboon, U. (1997). Roles of baffles in waste stabilization ponds. Water Science and Technology, 35(8), 275284. DOI: $10.1016 /$ S0273-1223(97)00177-7

Naddafi, K., Hassanvand, A. S., Dehghanifard, E., Faezi-Razi, D., Mostofi, S., Kasaee, N., Nabizadeh, R., \& Heidari, M. (2009). Performance evaluation of wastewater stabilization ponds in ArakIran. Journal of Environmental Health Science \& Engineering, 6(1), 41-46.

Oliveira, S. C., \& Sperling, M. V. (2011). Performance evaluation of different wastewater treatment technologies operating in a developing country. Journal of Water, Sanitation and Hygiene Development, 1(1), 37-56. DOI: 10.2166/washdev.2011.022

Olukanni, D. O. (2011). Hydraulic modeling and optimization of waste stabilization ponds design for developing nations (tesis doctoral). Covenant University, Nigeria.

Pedahzur, R., Nasser, A. M., Dor, I., Fattal, B., \& Shuval, H. I. (1993). The effect of baffle installation on the performance of a single-cell stabilization pond. Water Science and Technology, 27(7-8), 45-52.

Rolim, M. (2000). Sistemas de lagunas de estabilización, cómo utilizar aguas residuales tratadas en sistemas de regadío. Santa Fe de Bogotá, Colombia: Nomos.

Shilton, A. N., \& Harrison, J. (2003). Guidelines for the hydraulic design of waste stabilization ponds. Recuperado de http://www.bvsde.paho.org/bvsacd/agua2003/hidra 
Teçnología y

Ciencias $\stackrel{\Xi}{\unlhd}$ gua
2020, Instituto Mexicano de Tecnología del Agua

Open Access bajo la licencia CC BY-NC-SA 4.0

(https://creativecommons.org/licenses/by-nc-sa/4.0/)

Shilton, A. N., \& Mara, D. D. (2005). CFD (Computational Fluid Dynamics) Modeling of Baffles for Optimizing Tropical Waste Stabilization Ponds System. Water Science and Technology, 51(12), 103-106.

Sperling, M. V., Chernicharo, C. A., Soares, A. M., \& Zerbini, A. M. (2003). Evaluation and modelling of helminth eggs removal in baffled and unbaffled ponds treating anaerobic effluent. Water Science and Technology, 48(2), 113-120.

Swamee, P. K., \& Ojha, C. S. P. (1991). Drag coefficient and fall velocity of non-spherical particles. Journal of Hydraulic Engineering, $117(5), 660-667$.

Treviño-Cansino, A., \& Cortés-Martínez, F. (2016). Método de diseño reducido para lagunas de estabilización. Revista Mexicana de Ciencias Agrícolas, 7(4), 729-742.

Wood, M., Greenfield, P. F., Howes, T., Johns, M. R., \& Keller, J. (1995). Computational fluid dynamic modelling of waste stabilization ponds. Water Science and Technology, 31(12), 111-118.

Zanotelli, C. T., Medri, W., Belli-Filho, P., Perdomo, C. C., \& Costa, R. H. R. (2002). Performance of a baffled facultative pond treating piggery wastes. Water Science and Technology, 45(1), 49-53. 